\title{
BMJ Open Effectiveness and acceptability of myo- inositol nutritional supplement in the prevention of gestational diabetes (EMmY): a protocol for a randomised, placebo-controlled, double-blind pilot trial
}

Chiamaka Esther Amaefule, ${ }^{1}$ Zoe Drymoussi, ${ }^{1}$ Julie Dodds, ${ }^{1}$ Lorna Sweeney, ${ }^{2}$ Elena Pizzo, ${ }^{3}$ Jahnavi Daru, ${ }^{1}$ John Robson, ${ }^{4}$ Lucilla Poston, ${ }^{5}$ Asma Khalil, ${ }^{6}$ Jenny Myers, ${ }^{7}$ Angela Harden, ${ }^{2}$ Graham A Hitman, ${ }^{1}$ Khalid Khan, ${ }^{1}$ Javier Zamora, ${ }^{1,8}$ Mohammed S B Huda, ${ }^{9}$ Shakila Thangaratinam ${ }^{1}$

To cite: Amaefule CE, Drymoussi Z, Dodds J, et al. Effectiveness and acceptability of myo-inositol nutritional supplement in the prevention of gestational diabetes (EMmY): a protocol for a randomised, placebo-controlled, doubleblind pilot trial. BMJ Open 2018;8:e022831. doi:10.1136/ bmjopen-2018-022831

- Prepublication history and additional material for this paper are available online. To view these files, please visit the journal online (http://dx.doi. org/10.1136/bmjopen-2018022831).

Received 23 March 2018 Revised 4 July 2018 Accepted 31 July 2018

D) Check for updates

(c) Author(s) (or their employer(s)) 2018. Re-use permitted under CC BY-NC. No commercial re-use. See rights and permissions. Published by BMJ.

For numbered affiliations see end of article.

Correspondence to Miss Chiamaka

Esther Amaefule; c.e.amaefule@qmul.ac.uk

\section{ABSTRACT}

Introduction Gestational diabetes increases maternal and offspring complications in pregnancy and cardiovascular complications in the long term. The nutritional supplement myo-inositol may prevent gestational diabetes; however, further evaluation is required, especially in multiethnic high-risk mothers. Our pilot trial on myo-inositol to prevent gestational diabetes will evaluate trial processes, assess acceptability to mothers and obtain preliminary estimates of effect and cost data prior to a large full-scale trial.

Methods and analysis EMmY is a multicentre, placebocontrolled, double-blind, pilot, randomised trial, with qualitative evaluation. We will recruit pregnant women at $12-15^{+6}$ weeks' gestation, with gestational diabetes risk factors, from five maternity units in England between 2018 and 2019. We will randomise 200 women to take either $2 \mathrm{~g}$ of myo-inositol powder (intervention) or placebo, twice daily until delivery. We will assess rates of recruitment, randomisation, adherence to intervention and follow-up. Gestational diabetes will be diagnosed at 24-28 weeks as per the National Institute for Health and Care Excellence (NICE) criteria (fasting plasma glucose: $\geq 5.6 \mathrm{mmol} / \mathrm{L}$ and 2-hour plasma glucose: $\geq 7.8 \mathrm{mmol} / \mathrm{L}$ ). We will assess the effects of myo-inositol on glycaemic indices at 28 weeks and on other maternal, fetal and neonatal outcomes at postnatal discharge. Qualitative evaluation will explore the acceptability of the trial and the intervention among women and healthcare professionals. Cost data and health-related quality of life measures will be captured. We will summarise feasibility outcomes using standard methods for proportions and other descriptive statistics, and where appropriate, report point estimates of effect sizes (eg, mean differences and relative risks) and associated $95 \%$ Cls.

Ethics and dissemination Ethical approval was obtained through the London Queen Square Research Ethics Committee (17/L0/1741). Study findings will be submitted for publication in peer-reviewed journals. Newsletters will
Strengths and limitations of this study

Pilot study on trial processes, clinical outcomes and cost data to inform definitive trial.

- Qualitative evaluation on the acceptability of the trial and intervention.

- Pragmatically designed and reviewed by a patient and public involvement advisory group to allow for integration into current routine National Health Service clinical practice.

- Trial applicable only to women with proficiency in English language.

- Intervention available over the counter and may be accessible to trial participants.

be made available to participants, healthcare professionals and members of Katie's Team (a patient and public advisory group) to disseminate.

Trial registration number ISRCTN48872100.

Protocol version and date Version 4.0, 15 January 2018.

\section{INTRODUCTION}

Increasing rates of obesity worldwide, combined with sedentary lifestyle, has contributed to the rise in the number of women with gestational diabetes, a condition with high blood glucose levels diagnosed in pregnancy. Since 2010, the rates of gestational diabetes approach $24 \%$ in inner city maternity units in the UK. ${ }^{1}$ This is likely to be due to the multiethnic populations in inner city areas with high levels of 'at-risk' populations, who are at greater risk of gestational diabetes, such as women of South Asian origin . ${ }^{2}$ 
Gestational diabetes is associated with an increased risk of pregnancy complications including pre-eclampsia, macrosomia, caesarean section, postpartum haemorrhage, stillbirths and neonatal deaths. ${ }^{34}$ Therefore, pregnant women who are considered to be at high risk are offered a screening test for gestational diabetes. ${ }^{5}$ Pharmacological interventions such as metformin have not been shown to prevent gestational diabetes, and lifestyle interventions are challenging to implement, given their complexity. ${ }^{6}$ Myo-inositol, a nutritional supplement, has been reported to have beneficial effects in preventing gestational diabetes in some randomised trials. ${ }^{6}$

Existing randomised trials on myo-inositol are of poor quality, with small sample sizes, and involve homogeneous populations, mainly of Caucasian mothers from Italy. ${ }^{7}$ The generalisability of these findings to the National Health Service (NHS) setting is not known. Given the large sample size, and resources required to undertake a large-scale trial on the effects of myo-inositol on preventing gestational diabetes and its complications, there is a need to pilot trial procedures, ${ }^{8}$ ensure acceptability to participants and healthcare professionals and obtain relevant preliminary data.

\section{METHODS AND ANALYSIS \\ Study design}

$\mathrm{EMmY}$ is a multicentre, randomised, placebo-controlled, double-blind, pilot trial with a nested qualitative evaluation.

\section{Study aim and objectives}

The aim of the EMmY trial is to pilot study procedures and assess acceptability prior to undertaking a full-scale trial on myo-inositol supplementation during pregnancy to prevent gestational diabetes in high-risk women. Our primary objectives are to evaluate trial processes and procedures, obtain real-time data on the study design, assess adherence and report any side effects. Our secondary objectives are to assess the acceptability of the study and the intervention to pregnant women and healthcare professionals, identify reasons for non-participation and non-retention and identify barriers in recruitment and standardisation of care pathways for clinicians. Finally, we aim to obtain preliminary estimates on the effects of the intervention on glycaemic status, costs and quality of life measures.

\section{Study setting}

The $\mathrm{EMmY}$ trial will be conducted in five inner city maternity units including Barts Health Trust (The Royal London Hospital, Whipps Cross University Hospital and Newham University Hospital), St George's University Hospitals NHS Foundation Trust, and Manchester University Hospital NHS Foundation Trust (Manchester Royal Infirmary) over a period of 12 months (February 2018-January 2019).

\section{Study participants and eligibility criteria}

Pregnant women eligible for recruitment to the EMmY trial are those with a singleton, viable pregnancy from $12^{+0}$ to $15^{+6}$ weeks' gestation, able to provide written informed consent in English and with at least one of the following risk factors: family history of diabetes in any one of their first-degree relatives, gestational diabetes in a previous pregnancy, obesity (body mass index $\geq 30 \mathrm{~kg}$ / $\mathrm{m}^{2}$ ), minority ethnic family origin with a high prevalence of diabetes (such as South Asian and Black Caribbean/ African), polycystic ovary syndrome or previous macrosomic baby (birth weight $>4.5 \mathrm{~kg}$ ). Women on corticosteroids, metformin or insulin treatment are not eligible for recruitment. Women with known pre-existing type 2 diabetes or diagnosed with pregestational diabetes in early pregnancy will not be randomised. This will be based on first trimester glycated haemoglobin (A1c) (HbAlc) levels and/or fasting and 2 hour postprandial oral glucose tolerance test (OGTT) depending on individual Trust policy.

\section{Recruitment and randomisation}

All pregnant women booked for antenatal care will be screened against the eligibility criteria. Where possible, eligible participants will receive the EMmY Patient Information Sheet (PIS) at least 24 hours prior to their hospital booking visit or first trimester routine ultrasound scan depending on site policy. This is to make sure they have had ample time to consider the trial. The PIS will be accompanied by an invitation letter from the principal investigator (PI) informing patients that they may be approached by a member of the clinical research team, typically a research midwife to discuss participation in the trial at their hospital booking visit or first trimester ultrasound scan. The PIS will be discussed with eligible women by a member of the research team, typically a research midwife. Potential participants will have the opportunity to ask any questions and to clarify the study processes. If women are keen to join the study after these discussions, informed written consent will be obtained. Additional consent forms will be completed prospectively with regards to participating in qualitative interviews and the collection and storage of umbilical cord blood samples for future research. Women who do not wish to take part in the research interviews or consent to cord blood collection and storage can still participate in the trial. The informed written consent form is submitted as supporting information (see online supplementary file 1).

Following consent, participants with a history of gestational diabetes will undergo an $\mathrm{HbA1C}$ and/or an OGTT test depending on individual trust policy before 16 weeks' gestational age. These tests are conducted to rule out any potentially pre-existing but undiagnosed type 2 diabetes or early pregestational diabetes. Based on the National Institute for Health and Care Excellence (NICE) criteria, abnormal HbAlc results are defined as HbAlc levels $>48 \mathrm{mmol} / \mathrm{L}$ and abnormal OGTT results defined as a fasting blood glucose level $\geq 5.6 \mathrm{mmol} / \mathrm{L}$ and/or a 
2-hour blood glucose level $\geq 7.8 \mathrm{mmol} / \mathrm{L}$ post $75 \mathrm{~g}$ glucose load. ${ }^{5}$

Women with borderline HbA1c levels (41-47 mmol/L) at the booking visit will undergo first trimester OGTT. If the OGTT test shows an abnormal reading, these women will not be randomised. This process also allows us to assess the proportion of women with previous gestational diabetes, who enter subsequent pregnancies with potentially undiagnosed type 2 diabetes or pregestational diabetes. Participants eligible for randomisation will be randomly allocated to either the intervention group or the control group. The senior statistician will generate the allocation sequence. The randomisation and group allocation will be carried out through a secure online randomisation system. The randomisation scheme will be based on permuted blocks of random block size (sizes 4, 6 and 8 ), stratified by participating site. Research midwives will enrol and assign interventions to participants. Except for the senior statistician, study participants, care providers, data analysts and outcome assessors will be blinded to the group allocation. The research team will be unblinded only if necessary for the safety of the trial participant.

Following randomisation, baseline information on demographic and clinical characteristics will be collected from participants' maternity notes. The European Quality of Life 5-Dimensions 5-Level scale (EQ-5D-5L), a validated questionnaire, will be administered at baseline and at the end of the trial to capture quality-adjusted life-years (QALYs) in all participants. Participants in the intervention group will be provided with the myo-inositol powder supplement to be taken in a dose of $2 \mathrm{~g}$ twice daily from $12^{+0}$ to $15^{+6}$ weeks' gestational age until delivery. Participants in the control group will be provided with a placebo identical in colour, flavour and texture to the myo-inositol powder to be taken in the same dose and for the same duration of time. Information regarding supplement intake and dosage will be provided to prevent misinterpretation of instructions or ambiguity.

\section{Adherence to the intervention}

The participants will be provided with the intervention or placebo packs in two stages, with half of their supply being provided at visit 1 (recruitment and randomisation, at $12-15^{+6}$ gestational weeks) and the remaining half of their supply at visit 3 (approximately 28 gestational weeks). A paper-based diary and/or a mobile application (depending on participant's preference) will be provided to participants to self-report on adherence, with reminder features that we anticipate will encourage adherence. Participants will also be asked to bring any remaining sachets to their 28-week visit, where they will receive the next batch of intervention or placebo. A count of unused sachets (supplements) will be recorded as an additional measure of adherence to the intervention. Urinary inositol levels will also be tested at visit 3 (approximately 28 gestational weeks) as an additional measure of adherence in both groups. Text messages or phone calls will be made by the research team to remind participants of their upcoming appointments during the study. A participant will be deemed non-adherent if she has used $75 \%$ or less of her trial sachets. Figure 1 provides details of trial processes and procedures.

\section{Sample size calculation}

We expect that 1500 women will be booked for antenatal care each month at the participating hospitals and at least 300 of those will be eligible. Assuming 1000 eligible women were approached, we expect about $25 \%$ $(250 / 1000)$ to be consented. We expect that 20\% (50) of women who consent to the study will have a previous history of gestational diabetes. These women will undergo an early $\mathrm{HbA} 1 \mathrm{C}$ and/or an OGTT test before 16 weeks' gestational age to rule out any potentially pre-existing but undiagnosed type 2 diabetes or early pregestational diabetes. Any of these women with abnormal HbAlC $(>48 \mathrm{mmol} / \mathrm{L})$ and/or OGTT (fasting blood glucose $\geq 5.6 \mathrm{mmol} / \mathrm{L}$ and $/$ or a 2 -hour $75 \mathrm{~g}$ blood glucose level $\geq 7.8 \mathrm{mmol} / \mathrm{L}$ ) results and hence a diagnosis of early gestational diabetes will be excluded from the study. This will result in 200 women being randomised to either the myo-inositol or placebo arm. With an estimated attrition rate of $20 \%$, we expect that $160(160 / 200)$ women will remain in the study. Among these 160 women, we expect $80 \%$ of them $(128 / 160)$ to be adherent to the study processes. These numbers will allow for estimation of the 95\% CIs for trial feasibility outcomes with amplitudes of around $10 \%$.

\section{Primary and secondary outcome measures and outcome assessment}

The primary outcomes are the proportion of eligible, consented and randomised participants. The secondary outcomes include the acceptability of the study and the intervention as well as the proportion of outcome measures obtained in the trial. Laboratory outcomes will be assessed at 28 weeks' gestation including plasma glucose levels and the diagnosis of gestational diabetes. This will be achieved through fasting and 2-hour postprandial $75 \mathrm{~g}$ OGTT. Gestational diabetes will be diagnosed according to the 2015 NICE criteria (fasting glucose $\geq 5.6 \mathrm{mmol} / \mathrm{L}$, 2 hour $\geq 7.8 \mathrm{mmol} / \mathrm{L}$ ). Other laboratory outcomes include insulin levels, leptin and adiponectin levels, c-peptide levels at fasting and 2 hours postglucose load, (Homeostatic Model Assessment for Insulin Resistance (HOMAIR) and urinary inositol levels.

Maternal, fetal and neonatal outcomes will also be assessed at delivery and/or discharge. Maternal outcomes include pre-eclampsia, gestational age at delivery, postpartum haemorrhage, mode of delivery, preterm delivery before 34 and 37 weeks, perineal trauma, admission to high-dependency unit or intensive care unit, maternal death and maternal infection. Fetal and neonatal outcomes include, birth weight, macrosomia (birth weight $>4.5 \mathrm{~kg}$ ), admission to neonatal intensive care unit, shoulder dystocia, neonatal death, respiratory 


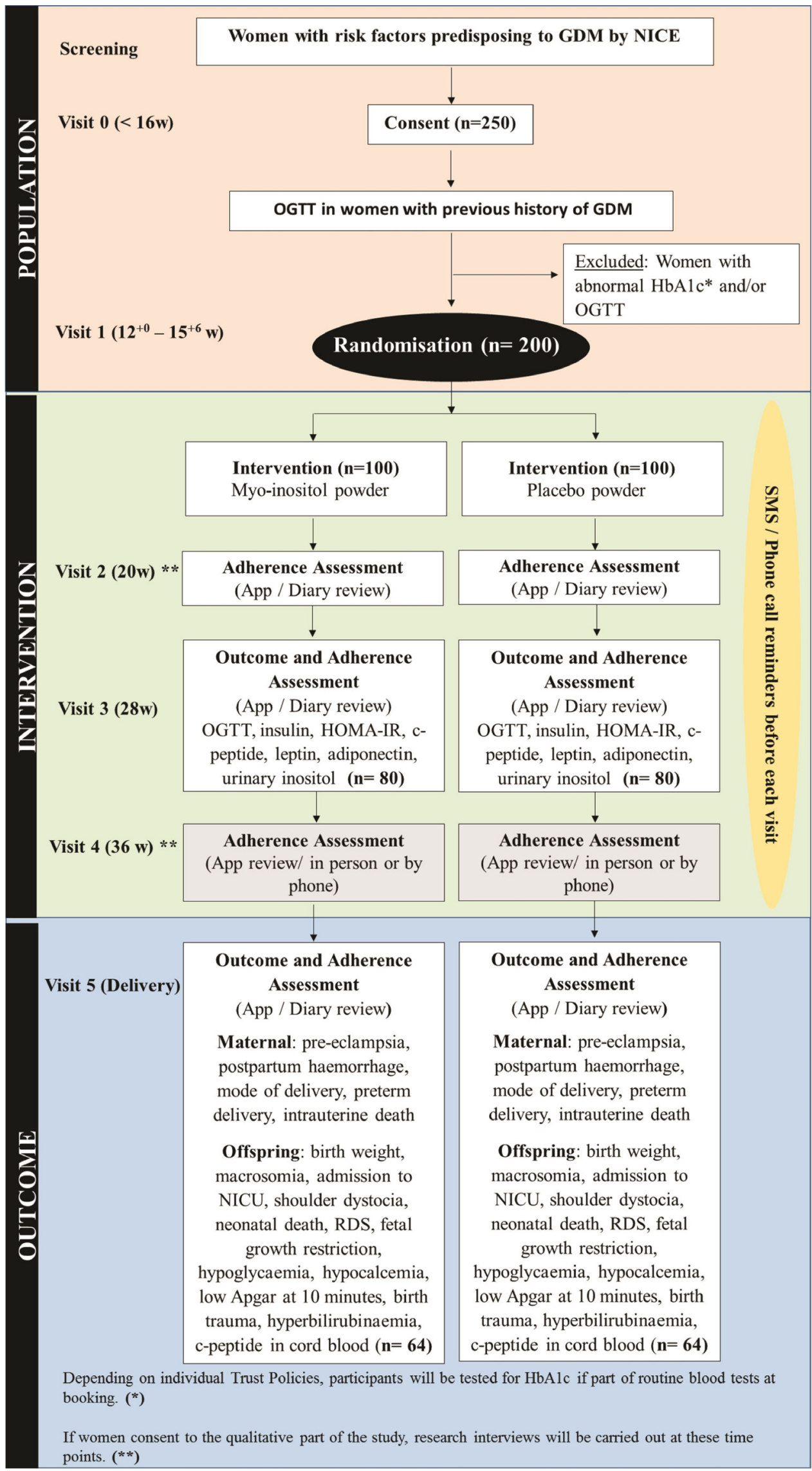

Figure 1 Trial scheme diagram on the conduct of the EMmY study. NICE, National Institute for Health and Care Excellence; NICU, neonatal intensive care unit; OGTT, oral glucose tolerance test; GDM, Gestational Diabetes Mellitus; HOMA-IR, Homeostatic Model Assessment for Insulin Resistance; RDS, Respiratory Distress Syndrome. 
Table 1 This table shows the schedule of assessments for the EMmY study

\begin{tabular}{|c|c|c|c|c|c|c|}
\hline Visit number & 0 & 1 & 2 & 3 & 4 & 5 \\
\hline Gestational weeks & $<16$ weeks & $\begin{array}{l}12- \\
15^{+6} \text { weeks }\end{array}$ & $\sim 20$ weeks & $\sim 28$ weeks & $\sim 36$ weeks & Delivery \\
\hline Consent & $x$ & & & & & \\
\hline Participant demographic data & $x$ & & & & & \\
\hline Randomisation & & $x$ & & & & \\
\hline Delivery of intervention & & $x$ & $x$ & $\mathrm{x}$ & $x$ & \\
\hline OGTT to diagnose GDM & & & & $x$ & & \\
\hline Assess adherence (app/diary) & & & $x$ & $x$ & $x$ & $x$ \\
\hline
\end{tabular}

*Assessments will be completed as near to the scheduled date as possible, depending on participant's appointment date. OGTT, oral glucose tolerance test.

distress syndrome, septicaemia, stillbirth, small for gestational age $\left(<10^{\text {th }},<5\right.$ th and $\left.<3^{\text {rd }}\right)$ as per population-based centile, hypoglycaemia, hypocalcaemia, Apgar at $10 \mathrm{~min}$, birth trauma such as shoulder dystocia fracture and hyperbilirubinaemia.

Cord blood will be collected and tested for c-peptide levels in the neonate. In addition, cost data and health-related quality of life (HRQL) measures will be captured. Cost data include the cost of myo-inositol and placebo administration, cost of routine tests, additional laboratory tests and other investigations in both groups, cost of clinic visits, hospital admissions, type of delivery, cost to treat adverse events (AEs), antenatal costs, postnatal costs and neonatal costs. Being a pilot trial, a sample size of 200 is adequate to assess feasibility outcomes but inadequate to show a difference in clinical outcomes between groups. However, we will be collecting these outcomes in this pilot trial to inform the future definitive full-scale trial on the feasibility of collecting and assessing these outcomes in our population groups.

A schedule of assessments is shown below in table 1 .

\section{Qualitative evaluation}

The qualitative evaluation will explore the acceptability of the trial and the intervention among participating women and healthcare professionals. This will be achieved through direct observation of recruitment and semistructured interviews as outlined below.

\section{Recruitment observation}

A sample of recruitment appointments (approximately 3-4 participants at each site) will be observed in order to gain detailed knowledge of women's specific needs or concerns, as well as factors in the recruitment setting/ environment that may impact on the recruitment process and outcomes.
Brief interview/open-ended questionnaire with those who decline recruitment

Women who decline to participate in the pilot trial will be invited to complete an open-ended questionnaire (either verbally or in writing) on: (1) their reasons for declining to participate and (2) their feedback on the recruitment process. Responses will inform recruitment procedures both for the pilot trial and the potential future full-scale trial. This model has been recently used in a pilot trial of group antenatal care at Barts Health NHS Trust (Pregnancy Circles study) and was found to work well as a means of identifying barriers to trial participation. ${ }^{9}$

\section{Interviews with randomised participants}

Following consent, semistructured interviews will be conducted with a purposive sample of approximately 15-20 women at different points in the trial. Interviews will be conducted approximately 2 months after randomisation (20-24 weeks of pregnancy) and towards the end of pregnancy (36-38 weeks) to capture women's experiences throughout pregnancy.

The first interview will explore participants' experience of their pregnancy so far, their understanding, beliefs and perception of gestational diabetes, perceived acceptability of the study procedures and intervention and other factors that can influence adherence. The second phase of interviews will purposively include 10 participants from the first interview phase who found intervention compliance and adherence particularly difficult or easy in the early stages of the study. This will be to address any further difficulties and supports for managing adherence to the intervention and any further experiences with study participation, data collection methods and follow-up procedures. We will also endeavour to interview a sample of women who drop out of the trial. 
All interviews will be audio-recorded with consent, and participants will also be offered a $£ 10$ voucher for each interview.

\section{Interviews and/or focus groups with healthcare professionals}

Semistructured interviews or focus groups will be conducted with a cross-section of key healthcare professionals (approximately 10-15 consisting of obstetricians, diabetologists and midwives) who are involved in delivering the intervention, and/or who have expertise in the area of gestational diabetes. Here, we will explore various approaches to managing/preventing GDM in multiethnic populations in the NHS setting. We will obtain their views on barriers to recruitment, compliance, retention and any suggestions on how these could be overcome. Informed consent will be gained, and a prepared interview guide will include questions arranged in topics.

\section{Patient and public involvement}

Prior to the grant application, the development of the EMmY research question was informed by patients' priorities and preferences. In our survey of pregnant women $(\mathrm{n}=71)$ within the Barts Health NHS Foundation Trust, $83 \%(59 / 71)$ agreed that there was a need to prevent gestational diabetes. More women indicated that they would be inclined to take myo-inositol $(79 \%, 56 / 71)$ than follow a complex lifestyle intervention $(60 \%, 43 / 71)$, and 8 out of 10 informed that they may or would definitely join a trial on myo-inositol. Additionally, two-thirds of healthcare professionals surveyed $(66 \%, 58 / 88)$ were keen to participate in a trial on myo-inositol.

We have collaborated with 'Katie's Team', a women's health and childbirth-specific patient and public involvement advisory group ${ }^{10}$ to inform several elements of the EMmY pilot trial. Katie's Team members contributed to the development of the study design, reviewed the trial documents such as the PISs and informed consent forms (ICFs) and developed the interview schedule. Patients and public representatives are not involved in recruitment or the conduct of the study.

We will submit study findings for publication in published in peer-reviewed journals. Study results will be circulated to participants, healthcare professionals and members of Katie's Team through newsletters, who in turn will further disseminate through traditional means and social media platforms.

\section{Data analysis}

\section{Statistical analysis}

Data will be analysed using descriptive statistics in order to inform trial feasibility and process. Given that this is a pilot study, the sample size is not adequately powered to assess the effect of the intervention on outcomes. However, where appropriate, we will present point estimates of effect sizes (eg, mean differences and relative risks) and associated 95\% CIs. The primary analysis will also involve the estimation of the proportions of the primary outcomes (ie, eligible women recruited into the trial, recruited women who complete the trial and adhere to the intervention treatment until delivery). We will also test if the proportions of the primary outcomes differ between the treatment group and the control group. We will explore the effect of mother's ethnicity, history of previous gestational diabetes and maternity unit attended on recruitment, adherence and attrition. All analysis will be performed using Stata software (StataCorp. 2015. Stata Statistical Software: Release V.14).

\section{Economic analysis}

A cost-utility analysis will be undertaken using a shortterm time horizon (the 'within trial' period) to obtain preliminary estimates of the cost-effectiveness of myo-inositol supplementation versus placebo in the prevention of gestational diabetes, which will inform the full-scale trial. The cost utility measures in the short run will be the incremental cost per unit of change per QALY gained. Unit costs will be collected and assessed from the perspective of the NHS and personal social services via standard sources. QALYs will be calculated based on the HRQL collected during the trial from the EQ-5D-5L questionnaires. The QALYs experienced from baseline to end of trial will be calculated as the area underneath this profile. Cost-utility will be calculated as the mean cost difference between the intervention and control group divided by the mean difference in outcomes to give the incremental cost-effectiveness ratio. Cost-effectiveness acceptability curves will be constructed, and we will subject the results to extensive deterministic sensitivity analysis.

\section{Qualitative data analysis}

Interview data will be subjected to thematic analysis. Transcripts will be coded for themes and concepts relating to women's and health professionals' experiences and perceptions of this intervention and the study. In this way, we will develop an analytical framework to identify key themes and how these inter-relate. Where possible, we will use constant comparison techniques and examine deviant cases to refine our analysis.

\section{Clinical management}

Besides myo-inositol and placebo, EMmY does not involve any other intervention. All aspects of antenatal care will be at the discretion of local clinicians. Further management of women diagnosed with gestational diabetes will be as per local guidelines for management of gestational diabetes.

\section{Participant withdrawal}

After consent, a participant can decide to self-withdraw from the trial. Clear distinctions will be made if the participant is only withdrawing from the trial but allowing further follow-up or withdrawing from both the trial and follow-up. A participant can also be withdrawn from the trial treatment if based on the opinion of the clinical carers and the investigators, it is medically necessary to do so. However, with any postrandomisation exclusions, the research team will endeavour to obtain and record 
the reasons for withdrawal and any AEs in the case report form (CRF). Where appropriate, efforts will be made to follow-up women who withdraw for all safety and efficacy outcomes.

If a participant explicitly withdraws consent to any further data collection, her decision will be respected, noted in the final study form and no further data will be collected from that participant. The participant will continue with NHS standard practice for follow-up care.

\section{Data management}

All participants in the $\mathrm{EMmY}$ trial will be given a unique trial number and will be identified to their local sites by their NHS hospital number. The chief investigator has a responsibility to ensure that participant anonymity is protected, maintained and associated participant information kept confidential and managed in accordance with the Data Protection Act (1998-UK), the sponsor's data management standard operating procedures (SOPs), The Research Governance Framework for Health and Social Care, The Research Ethics Committee Approval and the NHS Caldicott Guardian. All data will be monitored centrally and locally at the trials coordinating centre-Barts Research Centre for Women's Health (BARC) for consistency, viability and quality using bespoke data management systems.

All participants' data obtained for the trial, including personal information, will be anonymised, held securely and treated as strictly confidential. The data will be entered onto a secure computer database, either by a member of the research team or directly via a secure internet connection. All staff at each participating site and at the trials unit share a responsibility of care to prevent unauthorised disclosure of personal information. No identifiable individual data will be published. In accordance to the MRC guidelines on data retention, participants' collected data will be kept for 20 years following the end of trial to allow for verification and further data sharing via an individual patient data meta-analysis for instance.

\section{Monitoring and auditing}

The study sites will perform remote trial monitoring according to the agreed trial monitoring plan and self-monitoring template at the trials coordinating centre-BARC. Trial monitoring will include source data verification, checks on all ICFs and eligibility for randomisation log and a sample set of CRFs. Any major discrepancies with respect to trial regulatory matters and study protocol found at a site visit will trigger an audit of trial data by the coordinating team at the site involved, independently of the sponsor and investigators. The chief investigator will ensure that the trial is conducted in compliance with the principles of the Declaration of Helsinki (1996) and in accordance with all applicable regulatory requirements including, but not limited to, the Trust and Research Office policies and procedures, the Research Governance Framework, guidelines for good clinical practice and any subsequent amendments. Any form of non-compliance will be captured through communications and updates, monitoring visits, CRFs and other sources. In order to identify and verify any developing trends, the sponsor will maintain a log of any non-compliances, assess them and action a timeframe in which they need to be dealt with. In the event of any safety information that may require significant changes to the risk/benefit analysis of the study, the protocol, the ICF and the PIS will be amended and submitted to Research Ethics Committee (REC) for revision and approval. All participants of the EMmY study will be duly informed and provided with a revised copy of the PIS and the ICF to confirm their wish to continue where possible.

\section{Sample handling, labelling and logging}

Participants' samples collected will be processed by either NHS laboratory services, or by Affinity Biomarker Labs (Imperial College London). Samples will be labelled with the date of collection and participant's unique trial number. On arrival at the laboratories, samples will be handled as per routine clinical practice and local policies. Samples will be stored, processed and analysed by laboratory staff as defined by study SOPs, with any inconsistencies referred back to the research team or the clinical team. All samples received and processed in the NHS labs will be logged onto the NHS database, and samples received and processed by the Affinity Biomarker Labs will be logged onto the trial specific secure database.

\section{Trial organisation}

EMmY has a Project Steering Committee (PSC) that provides independent supervision of the trial, providing advice to the chief and coinvestigators and the sponsor on all aspects of the trial and affording protection for patients by ensuring the trial is conducted according to the principles of GCP in clinical trials. The trial will also be overseen by a Trial Management Group (TMG), who will meet regularly up until the end of the trial to evaluate trial progress and resolve any potential challenges. The TMG consists of the lead investigators, research midwives, and the project team at BARC.

$\mathrm{EMmY}$ is a pilot study with a low risk intervention and therefore no major safety concerns. In addition, EMmY aims to primarily assess the feasibility of conducting a potential full-scale trial rather than the effectiveness of the intervention and therefore does not require a data monitoring committee.

\section{Safety assessment}

Adverse events, are defined as any untoward medical occurrences in a participant that are not necessarily related to the intervention administered. AEs will be recorded by the PI in the CRF and the participant's medical notes. Participants experiencing AEs will be followed up by the research team. Serious adverse events (SAEs) are defined as any untoward occurrences that results in death, that is life threatening, requires hospitalisation or prolongation of existing hospitalisation, that results in persistent or significant disability or incapacity 
or is otherwise considered medically significant by the PI. Any SAEs will be reported to the sponsor within 24 hours of learning of the event and to the main REC within 15 days in line with the required timeframe. ${ }^{11}$

\section{Indemnity}

EMmY is sponsored by Queen Mary University of London (QMUL) as defined by the Research Governance Framework for Health and Social Care (April 2005). EMmY is also covered by the insurance brokers of QMUL on a 'No Faults Compensation for Clinical Trials and/or Human Volunteer Studies'. This policy covers or indemnifies the insured in respect of their legal liabilities arising out of the insured's activities.

\section{Ethics and dissemination}

EMmY has received site-specific approval for each participating site. EMmY is also registered online at ISRCTN. com (ISRCTN48872100). The chief investigator will coordinate dissemination of data from this study. The results from the trial will be submitted for publication in a major journal. The PSC will be responsible for approval of the main manuscript prior to submission for publication. Authorship of presentations and reports related to the study will be in the name of the lead investigators. Publications will name local coordinators as well as those involved in central coordination and trial management. The writing will be the responsibility of a writing committee including all of the investigators.

\section{DISCUSSION}

Approximately $40 \%$ of all women who are diagnosed with gestational diabetes progress to type 2 diabetes within 5 years postdelivery, in addition to their increased risk of gestational diabetes in future pregnancies. ${ }^{12} 13$ Infants born to mothers with gestational diabetes are at an increased risk of impaired glucose regulation, obesity and diabetes, leading to a vicious cycle of accumulated risks in the next generation. ${ }^{14} 15$ Therefore, preventing gestational diabetes provides intergenerational benefits, preventing chronic diseases in both mothers and their offspring.

With a projected increase in the NHS yearly spend (from $£ 8.8$ billion to $£ 13$ billion) on type 2 diabetes and its complications, ${ }^{16}$ preventing gestational diabetes has significant societal and economic benefits. However, evidence on effective and acceptable approaches to preventing gestational diabetes is lacking. ${ }^{17}$ Randomised trials on lifestyle changes has shown no significant difference in the incidence of gestational diabetes between groups. ${ }^{6} 18$ Women have reported on the difficulty of incorporating exercise into their daily routine as a result of child care, pregnancy symptoms and work commitments. ${ }^{17}$ Trials on the use of metformin in preventing the incidence of gestational diabetes also reported on no statistically significant difference between groups. ${ }^{18}$ There is therefore a need for a simple, effective, safe and acceptable intervention in preventing the onset of gestational diabetes in high-risk pregnant women.

Myo-inositol is a dietary nutritional supplement, which is present in staple foods such as meat and legumes, and is currently sold over the counter as a food supplement. Its use is not contraindicated in pregnancy. ${ }^{19}{ }^{20}$ Existing trials have shown the potential benefits of periconceptual myo-inositol supplementation in preventing folate resistant neural tube defects. The dose and timing of myo-inositol supplementation specified within the study are based on the need to ensure completion of fetal organogenesis by 12 weeks of gestation, reducing any theoretical risk to the fetus. ${ }^{21}$ Myo-inositol supplementation until the end of pregnancy also has a resulting effect on reducing macrosomia. ${ }^{22}$

Preliminary evidence on the effects of myo-inositol in pregnancy, based on small trials, suggests a reduction in gestational diabetes by up to $60 \% .^{6}$

To examine whether myo-inositol supplementation prevents the incidence of gestational diabetes in highrisk women, a large sample size will be required costing approximately $£ 1.7$ million. Prior to undertaking a largescale trial on the effects of myo-inositol, there is a need to pilot trial procedures, assess our ability to recruit and randomise women in a timely fashion and evaluate their adherence to study protocol and attrition rates. Evaluating adherence is vital to understanding women's perception of the intervention and the trial, as well as trial elements that may impact on their acceptability and hence retention in the trial. ${ }^{23}$ This may include women's understanding of gestational diabetes, their perception of risk, their attitudes towards a screening test for early pre-gestational diabetes and possible side effects of myo-inositol. It is important to explore potential variability across sites in the management of women at risk of gestational diabetes and subsequently women with a diagnosis of gestational diabetes. The knowledge of these factors can inform recruitment and intervention delivery strategies within the full-scale trial, allowing for the adaptation of trial processes to its local context being more sensitive to the needs of participants. ${ }^{24}$

\section{CONCLUSION}

The EMmY pilot trial is to inform a large definitive randomised controlled trial on the effects of myo-inositol supplementation on preventing the incidence of gestational diabetes and further complications in pregnant women at risk of developing gestational diabetes.

\section{Author affiliations}

${ }^{1}$ Barts Research Centre for Women's Health (BARC), Blizard Institute, Barts and the London School of Medicine and Dentistry, Queen Mary University of London, London, UK

${ }^{2}$ Institute for Health and Human Development, University of East London, London, London, UK

${ }^{3}$ Collaborations for Leadership in Applied Health Research and Care (CLAHRC) for North Thames London, Department of Applied Health Research, University College London, London, UK 
${ }^{4}$ Clinical Effectiveness Group, Barts and the London School of Medicine and Dentistry, Queen Mary University of London, London, UK

'Women's Health Unit, Guy's and St Thomas' NHS Foundation Trust, London, UK ${ }^{6}$ Department of Obstetrics and Gynaecology, St George's Hospital, London, UK ${ }^{7}$ Maternal \& Fetal Health Research Centre, University of Manchester, Manchester Academic Health Science Centre, Manchester, UK

${ }^{8}$ Clinical Biostatistics Unit (IRYCIS) and CIBER Epidemiology and Public Health, Madrid, Spain

${ }^{9}$ Department of Diabetes \& Metabolism, Barts Health NHS Trust, Royal London Hospital, London, UK

Acknowledgements The authors are thankful to the following organisations for their support and contributions towards the EMmY study: PharmaSure for the donation of myo-inositol and placebo and Montuno software for the discounted App. The authors are also thankful to the patient advisers for their contributions to the development of the study.

Contributors CEA: wrote the first draft of the manuscript, contributed to developing the qualitative evaluation plan of the study, will perform the qualitative data analysis and wrote the final version. ZD and JuD: contributed to drafting the manuscript, contributed to the methodology and logistics of the project and approved the final version. LS and AH: contributed to drafting the manuscript, developed the qualitative evaluation plan of the study, will supervise on the qualitative data analysis and has approved the final version of the manuscript. EP: contributed to drafting the manuscript, developed the economic evaluation plan, will lead the economic analysis and has approved the final version of the manuscript. JaD: contributed to drafting the manuscript, contributed to the methodology and logistics of the project, provided clinical input and has approved the final version. JR, LP, AK, JM, GAH, KK and MSBH: contributed to drafting the manuscript, provided clinical input and has approved the final version. JZ: contributed to drafting the manuscript, developed the statistical analysis plan, will lead the analysis and has approved the final version of the manuscript. ST: designed the project, developed the protocol, contributed to drafting the manuscript and approved the final version of the manuscript.

Funding This work was supported by Barts Charity, grant number MGU0373. The trial sponsor is Queen Mary University of London (sponsorsrep@bartshealth.nhs.uk). This research was supported by the National Institute for Health Research (NIHR) Collaboration for Leadership in Applied Health Research and Care North Thames at Barts Health NHS Trust (NIHR CLAHRC North Thames).

Disclaimer The funders and the trial sponsor have no role in the study conduct, dissemination and interpretation of results. The views expressed in this article are those of the author(s) and not necessarily those of the NHS, the NIHR, or the Department of Health and Social Care.

Competing interests None declared.

Patient consent Not required.

Ethics approval The London Queen Square Research Ethics Committee (17/ L0/1741).

Provenance and peer review Not commissioned; externally peer reviewed.

Open access This is an open access article distributed in accordance with the Creative Commons Attribution Non Commercial (CC BY-NC 4.0) license, which permits others to distribute, remix, adapt, build upon this work non-commercially, and license their derivative works on different terms, provided the original work is properly cited, appropriate credit is given, any changes made indicated, and the use is non-commercial. See: http://creativecommons.org/licenses/by-nc/4.0/.

\section{REFERENCES}

1. Farrar D, Simmonds M, Griffin S, et al. The identification and treatment of women with hyperglycaemia in pregnancy: an analysis of individual participant data, systematic reviews, meta-analyses and an economic evaluation. Health Technol Assess 2016;20:1-348.

2. Nishikawa E, Oakley L, Seed PT, et al. Maternal BMI and diabetes in pregnancy: Investigating variations between ethnic groups using routine maternity data from London, UK. PLoS One 2017; 12:e0179332.

3. Setji TL, Brown AJ, Feinglos MN. Gestational diabetes mellitus. Clinical diabetes, 2005:17-24.

4. Phillips PJ, Jeffries B. Gestational diabetes-worth finding and actively treating. Aust Fam Physician 2006;35:701-3.

5. National Institute for Health and Care Excellence. Diabetes in pregnancy: management from preconception to the postnatal period. 2015. NICE guideline [NG3] https://www.nice.org.uk/guidance/ ng3/chapter/1-Recommendations\#antenatal-care-for-women-withdiabetes-2 (accessed 11 Dec 2017).

6. Rogozińska E, Chamillard M, Hitman GA, et al. Nutritional manipulation for the primary prevention of gestational diabetes mellitus: a meta-analysis of randomised studies. PLoS One 2015;10:e0115526.

7. Crawford TJ, Crowther CA, Alsweiler J, et al. Antenatal dietary supplementation with myo-inositol in women during pregnancy for preventing gestational diabetes. Cochrane Database Syst Rev 2015;12:CD011507.

8. Craig P, Dieppe P, Macintyre S, et al. Developing and evaluating complex interventions: the new Medical Research Council guidance. BMJ 2008;337:a1655.

9. University College London. REACH study: Pregnancy circles pilot trial. 2017.

10. Moss N, Daru J, Lanz D, et al. Involving pregnant women, mothers and members of the public to improve the quality of women's health research. BJOG 2017;124:362-5.

11. The National Health Service Health Research Authority. Safety reporting. $2018 \mathrm{https} / / /$ www.hra.nhs.uk/approvals-amendments/ managing-your-approval/safety-reporting/ (accessed 13 Feb 2018).

12. Bellamy L, Casas JP, Hingorani AD, et al. Type 2 diabetes mellitus after gestational diabetes: a systematic review and meta-analysis. Lancet 2009;373:1773-9.

13. Schwartz N, Nachum Z, Green MS. The prevalence of gestational diabetes mellitus recurrence-effect of ethnicity and parity: a metaanalysis. Am J Obstet Gynecol 2015;213:310-7.

14. Herring SJ, Oken E. Obesity and diabetes in mothers and their children: can we stop the intergenerational cycle? Curr Diab Rep 2011;11:20-7.

15. Battista MC, Hivert MF, Duval K, et al. Intergenerational cycle of obesity and diabetes: how can we reduce the burdens of these conditions on the health of future generations? Exp Diabetes Res 2011;2011:1-19.

16. Hex N, Bartlett $C$, Wright $D$, et al. Estimating the current and future costs of Type 1 and Type 2 diabetes in the UK, including direct health costs and indirect societal and productivity costs. Diabet Med 2012;29:855-62.

17. Callaway LK, Colditz PB, Byrne NM, et al. Prevention of gestational diabetes: feasibility issues for an exercise intervention in obese pregnant women. Diabetes Care 2010;33:1457-9.

18. Madhuvrata P, Govinden G, Bustani R, et al. Prevention of gestational diabetes in pregnant women with risk factors for gestational diabetes: a systematic review and meta-analysis of randomised trials. Obstet Med 2015;8:68-85.

19. Holland \& Barrett Choline \& Inositol Caplets 500mg. http://www. hollandandbarrett.com/shop/product/holland-barrett-cholineinositol-caplets-500mg-60004280 (accessed 30 Oct 2014).

20. Livestrong.com. Is inositol safe while pregnant. http://www. livestrong.com/article/420380-is-inositol-safe-while-pregnant/ (accessed 10 December 2017).

21. Cavalli P, Tonni G, Grosso E, et al. Effects of inositol supplementation in a cohort of mothers at risk of producing an NTD pregnancy. Birth Defects Res A Clin Mol Teratol 2011;91:962-5.

22. D’Anna R, Scilipoti A, Giordano D, et al. Myo-inositol supplementation and onset of gestational diabetes mellitus in pregnant women with a family history of type 2 diabetes. Diabetes care 2013;36:854-7.

23. Hauser M, Lautenschlager M, Gudlowski Y, et al. Psychoeducation with patients at-risk for schizophrenia-an exploratory pilot study. Patient Educ Couns 2009;76:138-42.

24. Donovan J, Mills N, Smith M, et al. Quality improvement report: Improving design and conduct of randomised trials by embedding them in qualitative research: ProtecT (prostate testing for cancer and treatment) study. Commentary: presenting unbiased information to patients can be difficult. BMJ 2002;325:766-70. 